\title{
Evaluation of Energy Transfer between Grid Scale and Subgrid Scale by Use of Direct Numerical Simulation Data Base*
}

\author{
Toshio MIYAUCHI**, Mamoru TANAHASHI** \\ and Takashi KAKUWA**
}

\begin{abstract}
Energy transfer between grid scale(GS) and subgrid scale(SGS) induced by SGS stress in large eddy simulation is investigated by direct numerical simulation of homogeneous isotropic turbulence and a turbulent mixing layer. From the results of this study, the following conclusions were reached. (1) No correlation between the Leonard term and Cross term exists from the viewpoint of energy transfer. (2) The Smagorinsky model for the Reynolds term predicts very large energy transfer compared with that obtained from the DNS data. (3) The Bardina models for the Reynolds term and Cross term predict large energy transfer from SGS to GS in the high wave number region, which indicates that the Bardina model causes excessive backward scattering in the high wave number region.
\end{abstract}

Key Words: Computational Fluid Dynamics, Numerical Analysis, Large Eddy Simulation, Shear Flow, Energy Transfer Function, Subgrid Scale Model, Direct Numerical Simulation

\section{Introduction}

Numerical simulations are extremely important for estimation and prediction of turbulent flows in engineering applications. In recent years, direct numerical simulation(DNS) in which Navier-Stokes equations are solved without turbulence models has become practical due to the progress achieved in supercomputer development. However, restrictions in CPU time and memory capacity do not allow the use of DNS for the analysis of flow fields in engineering applications. The flow fields that can be analyzed by DNS are limited to simple geometry flows, such as channel flow, homogeneous sheer flow and a turbulent mixing layer, and low Reynolds number flows.

Large eddy simulation(LES) makes it possible to solve the turbulent flows with high Reynolds number that commonly appear in engineering applications. In LES, the grid scale(GS) component that can be

* Received 22nd November 1996. Japanese original: Trans. Jpn. Soc. Mech. Eng., Vol.62, No. 596, B (1996), p. 1406-1413 (Received 7th May 1995)

** Department of Mechano-Aerospace Engineering, Tokyo Institute of Technology, 2-12-1 Ookayama, Meguro-ku, Tokyo 152, Japan resolved by the computational grid is directly calculated, and fluctuations smaller than the grid scale are modeled by use of a subgrid scale(SGS) model. Usually the Smagorinsky model ${ }^{(1)}$ and Bardina model $^{(2)}$ are used to close the governing equations for the GS component. The Smagorinsky and Bardina models are constructed in physical space. On the other hand, there are spectrum eddy viscosity model $^{(3),(4)}$. in which the energy transfer function ${ }^{(5)}$ is evaluated for construction of a SGS stress model. However, it is difficult to apply these models to the complex flows which must be solved by finite difference schemes.

The objective of this study is to evaluate the Smagorinsky and Bardina models in Fourier space in terms of the energy transfer between GS and SGS, and to obtain insight into possible means of improvement of SGS models. DNS data bases of incompressible homogeneous isotropic turbulence and a turbulent mixing layer are used to evaluate the energy transfer between GS and SGS.

\section{Nomenclature}

$C_{S}$ : Model constant of Smagorinsky model $C_{B}$ : Model constant of Bardina model 
$C_{i j}:$ Cross term

$E$ : Energy spectrum

$k_{g}$ : SGS energy

$k_{x}$ : Wave number in the streamwise direction

$k_{y}$ : Wave number in the transverse direction

$k_{z}$ : Wave number in the spanwise direction

$L_{i j}:$ Leonard term

$R e$ : Reynolds number

$R_{i j}$ : Reynolds term

$t$ : Nondimensional time

$T$ : Energy transfer function

$T_{L}$ : Energy transfer by Leonard term

$T_{c}$ : Energy transfer by Cross term

$T_{R}$ : Energy transfer by Reynolds term

$T_{S G S}$ : Energy transfer between GS and SGS

$u_{i}$ : Velocity component in the $i$ direction

$U_{0}$ : Mean velocity difference across a mixing layer

$x:$ Coordinate in the streamwise direction

$y$ : Coordinate in the transverse direction

$z$ : Coordinate in the spanwise direction

$\delta$ : Momentum thickness of a mixing layer

$\delta_{\omega}$ : Initial vorticity thickness of a mixing layer

$\delta_{i j}$ : Delta function

$\Delta:$ Width of a filter

$\lambda$ : The most unstable wavelength for an initial mean velocity profile

$\nu$ : Kinematic viscosity

$\tau_{i j}:$ Total SGS stress

Symbols

: Fourier coefficient

- : Grid-scale variable

': Subgrid-'scale variable

*: Complex conjugate

$\sim$ : Fourier coefficient defined by Eq. (22)

\section{DNS Data Bases}

\section{1 Homogeneous isotropic turbulence}

The DNS data base of homogeneous isotropic turbulence is constructed by use of a Fourier spectral method with $96 \times 96 \times 96$ grid points. The computational conditions are selected to satisfy the experiment conditions described by Comte-Bellot and Corssin ${ }^{(6)}$. The Reynolds number based on the Taylor microscale is 37.2. The results of the DNS coincide with the experimental results excellently for all turbulent statistics. Figure 1 shows the energy spectrum at the time when the SGS model was evaluated. The exact energy transfer calculated from the DNS data is compared with the energy transfer calculated using the SGS models. For the filter operation, a Gaussian filter corresponding to $48 \times 48 \times 48$ grid points and a sharp cut-off filter with cut-off wave number $k_{c}$ are applied to the DNS data.

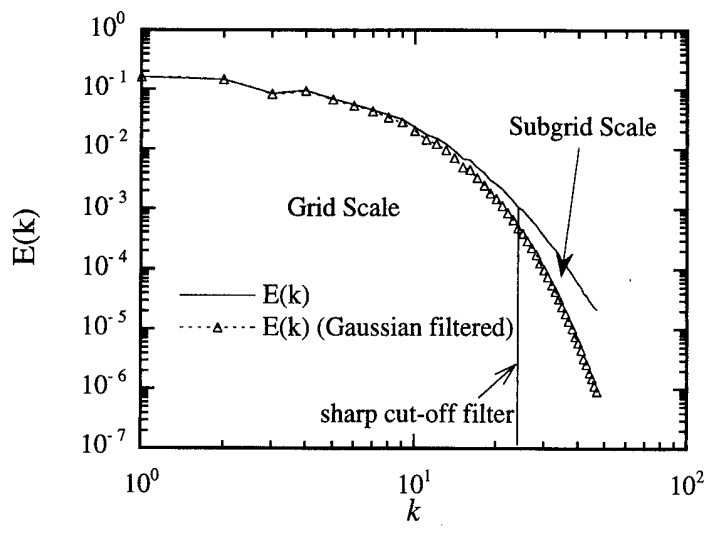

Fig. 1 Energy spectrum of a DNS data base of homogeneous isotropic turbulence

\subsection{Turbulent mixing layer}

The Reynolds number of the DNS data base of a turbulent mixing layer is $600^{(7)}$. The Reynolds number is defined by an initial vorticity thickness and the mean velocity difference. The calculation region is set equal to $2 \lambda \times 4 \lambda \times 4 / 3 \lambda(\lambda=7.078)$ in the streamwise, transverse and spanwise directions, respectively, where $\lambda$ is the most unstable wavelength for an initial mean velocity profile. The calculation is conducted at $120 \times 241 \times 80$ grid points by use of a spectral method. It has been reported that the calculated turbulent mixing layer is fully developed and composed of many fine-scale vortex tubes ${ }^{(7)}$. The GS-SGS energy transfer is investigated by use of the data base at $t=80$. The Reynolds number based on momentum thickness at $t=80$ is 702 , and the visual Reynolds number is about 6000 . For the filter operation, a Gaussian filter corresponding to $30 \times 61 \times 20$ grid points and a sharp cut-off filter are applied to the DNS data.

\section{Energy Transfer Function and SGS Models}

By application of a filter to the Navier-Stokes equations and continuity equation, the following governing equations for GS components are obtained:

$$
\begin{aligned}
& \frac{\partial \bar{u}_{i}}{\partial t}+\bar{u}_{j} \frac{\partial \bar{u}_{i}}{\partial x_{j}}=-\frac{\partial \bar{p}}{\partial x_{i}}-\frac{\partial \tau_{i j}}{\partial x_{j}}+\nu \frac{\partial^{2} \bar{u}_{i}}{\partial x_{j} \partial x_{j}}, \\
& \frac{\partial \bar{u}_{i}}{\partial x_{i}}=0
\end{aligned}
$$

where the overbar denotes a grid-scale variable and $\tau_{i j}$ is the SGS stress. This SGS stress can be separated into the Leonard term $L_{i j}$, Cross term $C_{i j}$ and Reynolds term $R_{i j}$ as

$$
\begin{aligned}
& \tau_{i j}=L_{i j}+C_{i j}+R_{i j}, \\
& L_{i j}=\bar{u}_{i} \bar{u}_{j}-\bar{u}_{i} \bar{u}_{j}, \\
& C_{i j}=\bar{u}_{i} u_{j}^{\prime}+\bar{u}_{i}^{\prime} \bar{u}_{j}, \\
& R_{i j}=\bar{u}_{i}^{\prime} u_{j}^{\prime} .
\end{aligned}
$$

These SGS stresses represent the energy transfer between the GS component and SGS component. Evaluation of the SGS models in terms of the GS-SGS 
energy transfer is very important for highly accurate prediction of turbulent flows.

Here, we consider the energy transport in Fourier space. From the continuity equation and NavierStokes equations, the following transport equation for the energy $E(k)$ can be obtained:

$$
\frac{\partial E(k)}{\partial t}=T(k)-2 \nu k^{2} E(k)
$$

where $T(k)$ is the energy transfer function and is given $\mathrm{as}^{(5)}$

$$
\begin{aligned}
& T(k)=\frac{1}{2} \operatorname{Im}\left\{\sum_{k-1 / 2 \leq|\boldsymbol{k}|<k+1 / 2} P_{\alpha \beta \gamma}(\boldsymbol{k})\right. \\
& \left.\quad \times \sum_{\boldsymbol{k}=\boldsymbol{p}+\boldsymbol{q}} \widehat{u}_{\alpha}^{*}(\boldsymbol{k}) \widehat{u}_{\beta}(\boldsymbol{k}) \widehat{u}_{\gamma}^{*}(\boldsymbol{k})\right\}, \\
& P_{\alpha \beta \gamma}(\boldsymbol{k})=k_{\beta}\left(\delta_{\alpha \gamma}-\frac{k_{\alpha} k_{\gamma}}{|\boldsymbol{k}|^{2}}\right)+k_{\gamma}\left(\delta_{\alpha \beta}-\frac{k_{\alpha} k_{\beta}}{|\boldsymbol{k}|^{2}}\right) .
\end{aligned}
$$

In Eq. ( 8), the asterisk means complex conjugate. Similarly, the equation for GS energy can be obtained from Eqs. (1) and (2) as

$$
\begin{aligned}
& \frac{\partial \bar{E}(k)}{\partial t}=\bar{T}(k)-T_{S G S}(k)-2 \nu k^{2} \bar{E}(k), \\
& T_{S G S}(k)=\sum_{k-1 / 2 \leq|\boldsymbol{k}|<k+1 / 2} \hat{\bar{u}}_{i}^{*}(\boldsymbol{k})\left[i k_{j} \hat{\tau}_{i j}(\boldsymbol{k})\right],
\end{aligned}
$$

where $T_{S G S}(k)$ corresponds to the SGS stress term in the physical space. If a sharp cut-off filter with cutoff wave number $k_{C}$ is used in the filter operation, $T_{S G S}(k)$ can be obtained as $T_{S G S}(k)=T\left(k, k_{C}\right)-T(k)$. $T\left(k, k_{c}\right)$ can be defined from Eq. ( 8$)$ with additional conditions of $|k|,|p|,|q|<k_{C}$. In this study, $T_{S G S}(k)$ is used to evaluate the SGS models. $T_{S G S}(k)$ can be separated into three terms corresponding to a Leonard term, Cross term and Reynolds term as

$$
\begin{aligned}
& T_{S G S}(k)=T_{L}(k)+T_{C}(k)+T_{R}(k), \\
& T_{L}(k)=\sum_{k-1 / 2 \leq|\boldsymbol{k}|<k+1 / 2} \hat{\bar{u}}_{i}^{*}(\boldsymbol{k})\left[i k_{j} \hat{L}_{i j}(\boldsymbol{k})\right], \\
& T_{C}(k)=\sum_{k-1 / 2 \leq|\boldsymbol{k}|<k+1 / 2} \hat{\bar{u}}_{i}^{*}(\boldsymbol{k})\left[i k_{j} \hat{C}_{i j}(\boldsymbol{k})\right], \\
& T_{R}(k)=\sum_{k-1 / 2 \leq|\hat{k}|<k+1 / 2} \hat{\bar{u}}_{i}^{*}(\boldsymbol{k})\left[i k_{j} \hat{R}_{i j}(\boldsymbol{k})\right],
\end{aligned}
$$

where positive value of $T_{S G S}(k), T_{L}(k), T_{C}(k)$ and $T_{R}(k)$ indicates that the energy is transferred from the GS component to the SGS component, and negative value indicates the reverse energy transfer.

\section{Sharp Cut-Off Filter and Energy Transfer}

In this section, effects of a sharp cut-off filter on the GS-SGS energy transfer are discussed in the case of homogeneous isotropic turbulence. Figure 2 shows the eddy viscosity coefficients in the wave number space calculated from $T_{S G S}(k)$ by application of a sharp cut-off filter. Here, the eddy viscosity coefficient is defined $\mathrm{as}^{(3),(4)}$

$$
\nu_{e}\left(k / k_{c}\right)=\frac{T\left(k, k_{c}\right)-T(k)}{2 k^{2} E(k)} .
$$

The eddy viscosity coefficient depends on the cut-off

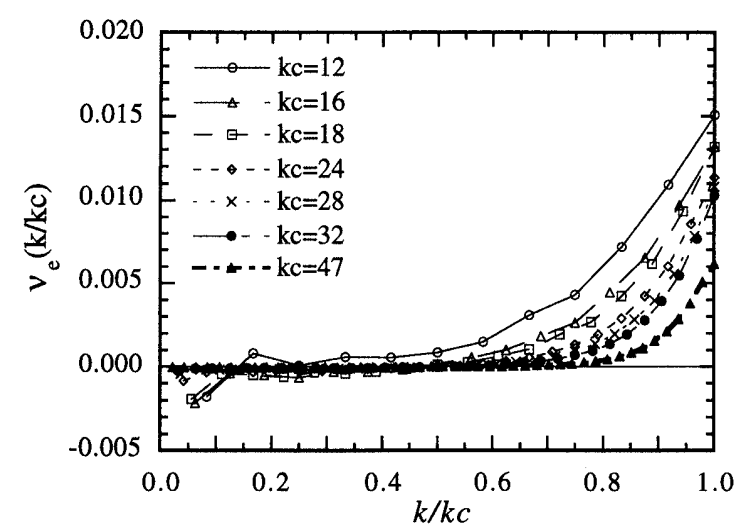

Fig. 2 Eddy viscosity coefficient calculated from DNS data of homogeneous isotropic turbulence by application of a sharp- ${ }^{-}$cutoff filter

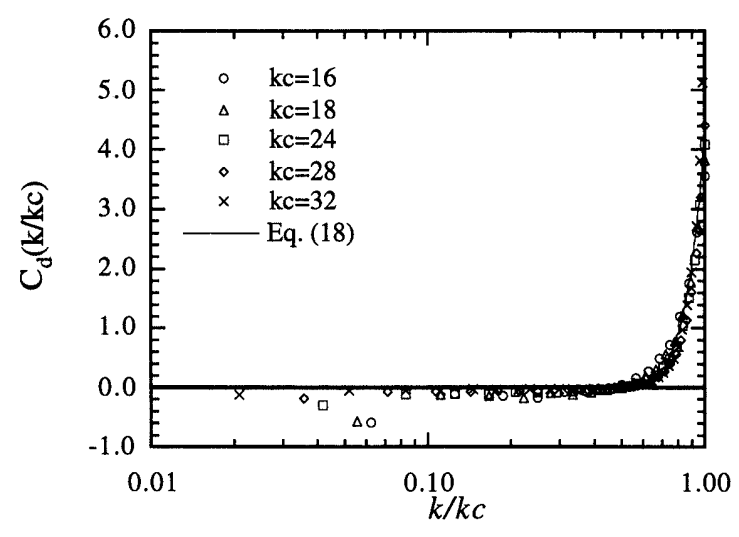

Fig. 3 Normalized eddy viscosity coefficient

wave number and becomes very large with decrease of the cut-off wave number. This characteristic becomes very important in the following sections where a Gaussian filter and a cut-off filter are applied together.

Figure 3 shows eddy viscosity coefficient normalized as follows:

$$
\begin{aligned}
& C_{d}=\nu_{e}\left(k / k_{c}\right)\left[k_{c} / E\left(k_{c}\right)\right]^{1 / 2}\left[k_{c} / k_{d}\right]^{-2}, \\
& C_{d}=2.4 \times 10^{3} \exp \left(-6.4 k_{c} / k\right),
\end{aligned}
$$

where $k_{d}$ is the Kolmogorov wave number. It is clear that the eddy viscosity can be normalized by Eq. (17) and approximated by Eq. (18). However, it is difficult to apply the eddy viscosity represented in the Fourier space to LESs of complex turbulent flows which is important in the engineering applications.

\section{Evaluation of the SGS Models Based on Energy Transfer}

\subsection{GS-SGS energy transfer in homogeneous isotropic turbulence}

In this section, we will discuss the GS-SGS energy transfer in homogeneous isotropic turbulence. Figure 4 shows the exact $T_{L}(k), T_{C}(k)$ and $T_{R}(k)$ obtained from the DNS data base of homogeneous isotropic 
turbulence by application of a Gaussian filter. The Cross term shows a large value in the low-wave number region and the Leonard term shows a large value in the middle wave number region. Previously, it has been reported that the coefficient of correlation between these two terms is nearly -1 in physical space $^{(7)-(9)}$. However, from the viewpoint of the energy transfer, these two terms show almost no correlation. Compared with the Leonard and Cross terms, the Reynolds term shows a small energy transfer, because the Reynolds number of the DNS data base is relatively low. Figure 5 shows the energy transfer obtained by use of the Bardina model for the Cross term ${ }^{(2)}$

$$
C_{i j}=\overline{\bar{u}}_{j}\left(\bar{u}_{i}-\overline{\bar{u}}_{i}\right)+\left(\bar{u}_{j}-\overline{\bar{u}}_{j}\right) \overline{\bar{u}}_{i}
$$

It has been shown that the Bardina model gives an accurate estimation of the Cross term ${ }^{(2),(7)-(9)}$. Figure 5 suggests that the overall feature of the energy transfer in the relatively low wave number region coincides with that indicated by the DNS data. The energy transfer obtained form the DNS data base starts from a large positive value in the low wave

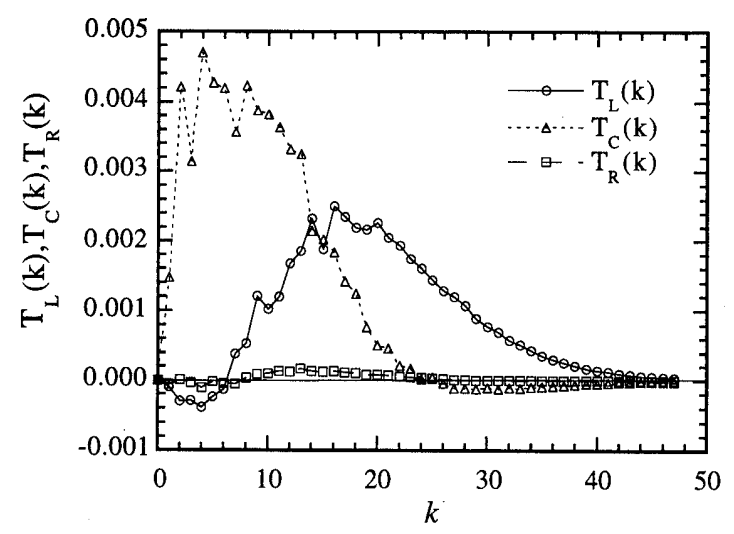

Fig. 4 Energy transfer between GS and SGS in homogeneous isotropic turbulence in the case of a Gaussian filter

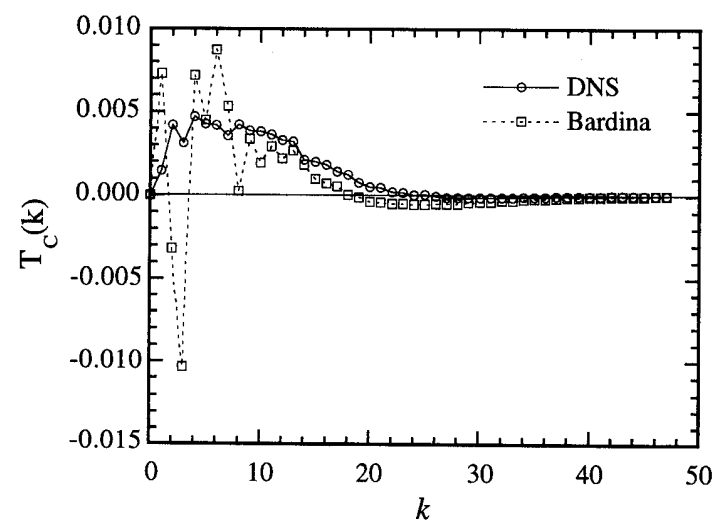

Fig. 5 GS-SGS energy transfer induced by the Cross term in homogeneous isotropic turbulence in the case of a Gaussian filter number region and approaches zero with increasing wave number. However, $T_{c}(k)$ of the Bardina model shows a negative value from $k=19$, which means that energy is transferred from the SGS components to the GS components in the high wave number region. This may result in the formation of unphysical small-scale fluctuations in LES and lead to numerical instability. In fact, this phenomenon is one of the reasons why the small-scale vortices are created in a dynamic test of the Bardina model in a turbulent mixing layer.

Figure 6 shows the energy transfer obtained by use of the Smagorinsky model ${ }^{(1)}$ and Bardina model $^{(2),(10)}$ for the Reynolds term. These models are represented as

$$
\begin{aligned}
& R_{i j}=\frac{2}{3} k_{g} \delta_{i j}-2\left(C_{S} \Delta\right)^{2} \bar{S} \bar{S}_{i j}, \bar{S}=\left(2 \bar{S}_{i j} \bar{S}_{i j}\right)^{1 / 2}, \\
& R_{i j}=C_{B}\left(\bar{u}_{i}-\overline{\bar{u}}_{i}\right)\left(\bar{u}_{j}-\overline{\bar{u}}_{j}\right),
\end{aligned}
$$

where $k_{g}$ denotes. GS energy. Both models predict the general behavior of the energy transfer. However, in the case of the Smagorinsky model, the peak of the energy transfer is shifted to the low wave number region and the absolute value is very large. Here, the model constant for the Smagorinsky model $C_{S}$ is set equal to $0.2^{(11)}$, and that for the Bardina model $C_{B}$ is $4.5^{(7),(11)}$. As shown for the Cross term, the sign of energy transfer expected from the Bardina model changes at $k=23$. The magnitude of this negative energy transfer is more significant for the Reynolds term than for the Cross term.

In the above, a Gaussian filter was used for the filter operation. In a real LES, however, a Gaussian filter and a sharp cut-off filter must be used together. Next, the energy transfer in the case of a Gaussian filter and a sharp cut-off filter will be discussed. Figure 7 shows the exact energy transfer calculated from the DNS data. As expected from the case with a sharp cut-off filter(Fig. 2), the energy transfers in the double filter case become larger than that in the

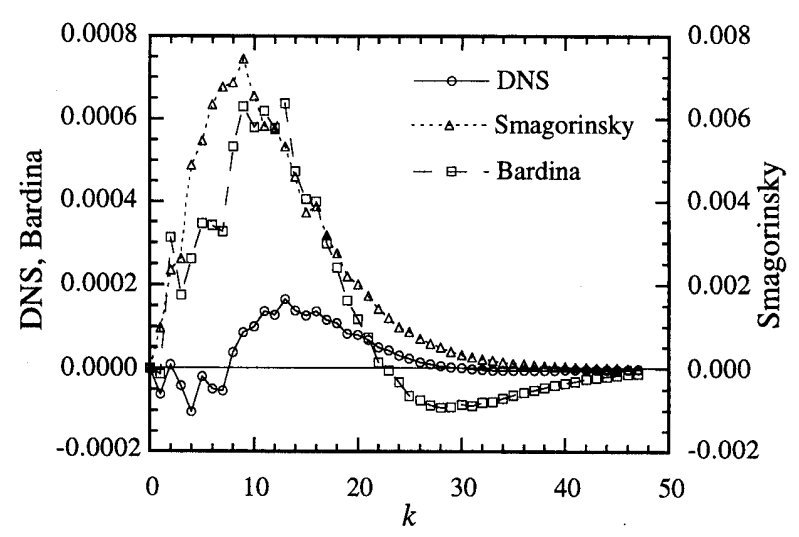

Fig. 6 GS-SGS energy transfer induced by the Reynolds term in homogeneous isotropic turbulence in the case of a Gaussian filter 
case of the single Gaussian filter near the cut-off wave number.

Figure 8 shows the energy transfer obtained by use of the Bardina model for the Cross term. $T_{c}(k)$ of the Bardina model shows a negative value in the high wave number region and the energy is transferred to

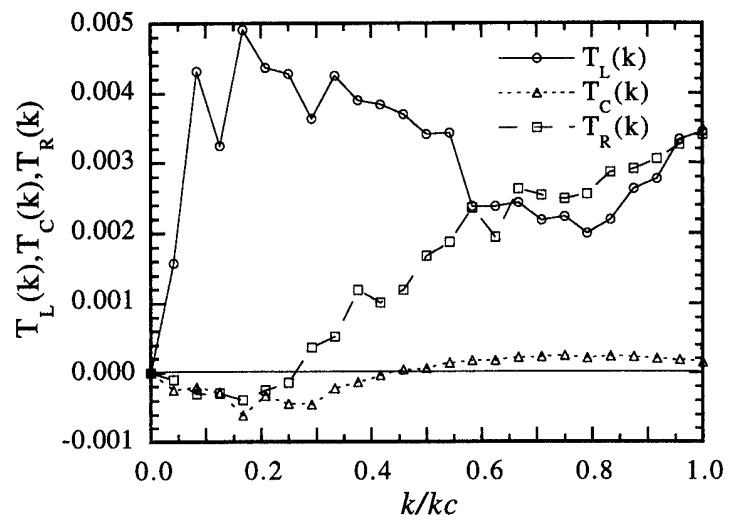

Fig. 7 Energy transfer between GS and SGS in homogeneous isotropic turbulence in the case of a Gaussian filter and a sharp cut-off filter

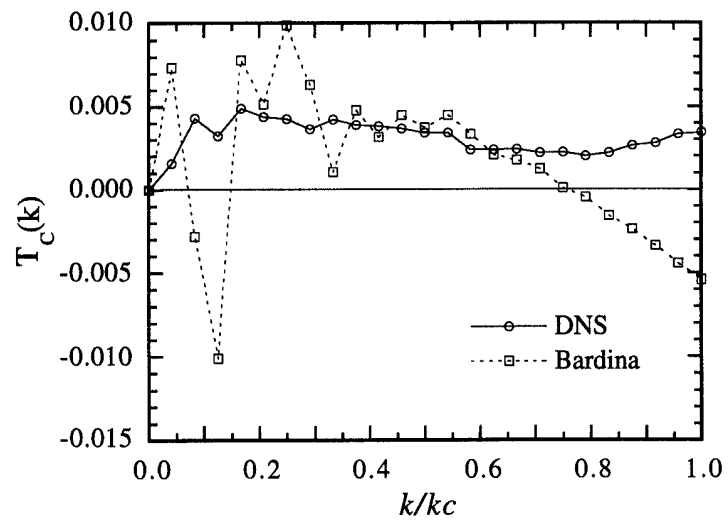

Fig. 8 GS-SGS energy transfer induced by the Cross term in homogeneous isotropic turbulence in the case of a Gaussian filter and a sharp cut-off filter

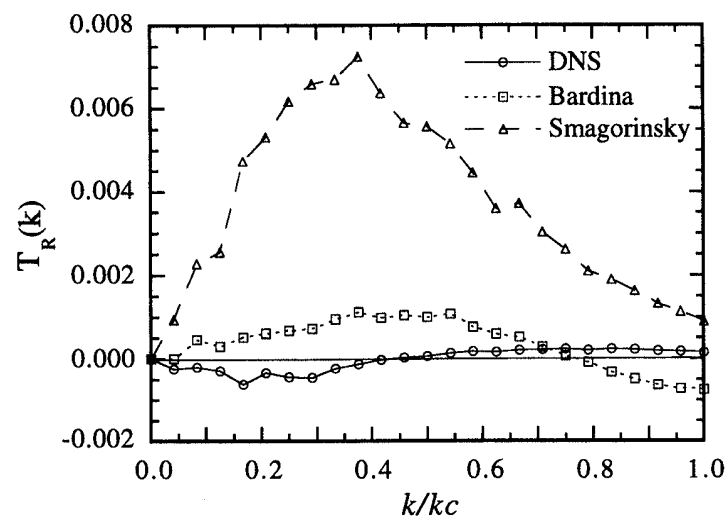

Fig. 9 GS-SGS energy transfer induced by the Reynolds term in homogeneous isotropic turbulence in the case of a Gaussian filter and a sharp cut-off filter the small-scale fluctuations of the GS component. This result is similar to that in the case of the single Gaussian filter, while the difference between the exact energy transfer and the predicted one becomes large because the energy transfer in the high wave number region is pronounced due to the sharp cut-off. Figure 9 shows the same figure for the Reynolds term. The Smagorinsky and Bardina models show positive values in the low wave number region, which are different from the DNS data that show negative values for $k / k_{C}<0.4$. In the case of the Bardina model, an inverse energy transfer is also predicted to occur in the high wave number region.

\section{2 GS-SGS energy transfer in a turbulent mixing layer}

In homogeneous isotropic turbulence, the physical properties are isotropic and it is possible to assume periodicity in all directions. However, in a temporally developing turbulent mixing layer, there are only two directions that can be assumed to be homogeneous. Therefore, we modified the energy transfer between GS and SGS in the turbulent mixing layer. We apply the following transformations on each $x-z$ plane horizontal to the mean shear direction:

$$
\begin{aligned}
& u_{i}(x, y, z, t)=\sum_{k_{x}=-N_{x} / 2}^{N_{x} / 2-1} \sum_{k_{z}=-N_{z} / 2}^{N_{z} / 2-1} \tilde{u}_{i}\left(k_{x}, y, k_{z}, t\right) \\
& \quad \times \exp \left(i k_{x} x+i k_{z} z\right) .
\end{aligned}
$$

Applying similar transformations to Eqs. (1) and ( 2 ), we can construct an energy equation for the GS component defined on each $x-z$ plane as

$$
\frac{\partial \bar{E}(k, y)}{\partial t}=N(k, y)-T_{S G S}(k, y)+\nu D(k, y)
$$

where

$$
\begin{aligned}
& T_{S G S}(k, y)=T_{L}(k, y)+T_{C}(k, y)+T_{R}(k, y), \\
& k=\sqrt{k_{x}^{2}+k_{z}^{2}} .
\end{aligned}
$$

The GS-SGS energy transfer can be separated into a Leonard term, Cross term and Reynolds term similar to the case of homogeneous isotropic turbulence.

Figure 10 shows the energy spectrum on the $x-z$

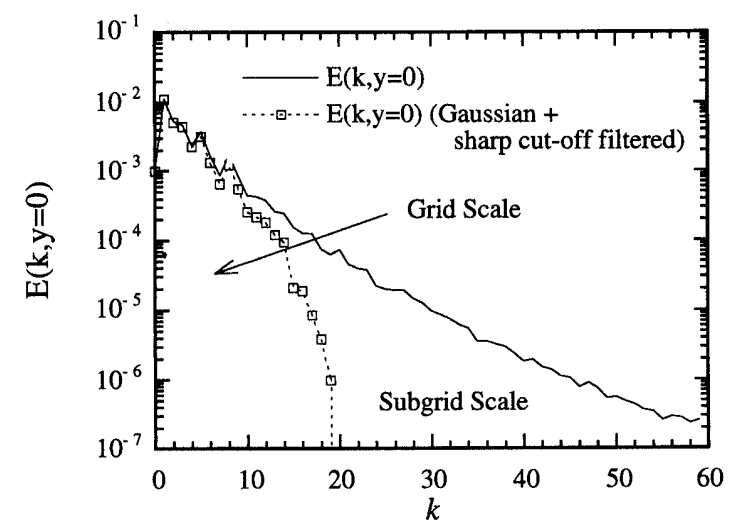

Fig. 10 Energy spectrum of a DNS data base of a turbulent mixing layer at $y=0$ 
plane with $y=0$ and the relation between the GS and SGS components in the case of a Gaussian filter with the grid points $30 \times 61 \times 20$. In the case of the turbulent mixing layer, we will discuss the case of a Gaussian filter and a sharp cut-off filter. Figures 11 and 12 show the energy transfer between GS and SGS energy obtained from the DNS data in the center of the shear layer $(y=0)$ and in the intermittent region of the outer

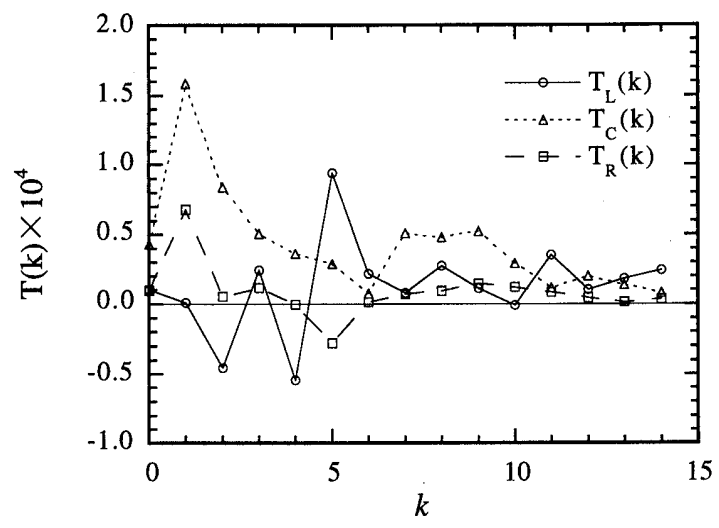

Fig. 11 GS-SGS energy transfer at $y=0$ in a turbulent mixing layer

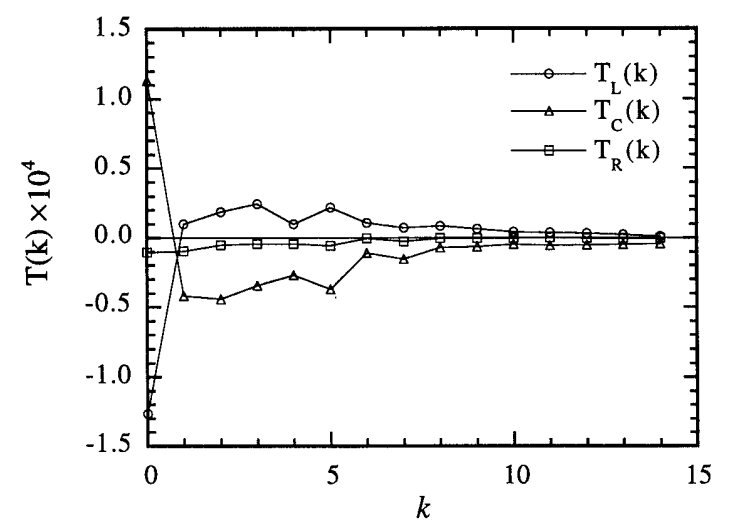

Fig. 12 GS-SGS energy transfer at $y=-2 / 3 \lambda$ in a turbulent mixing layer

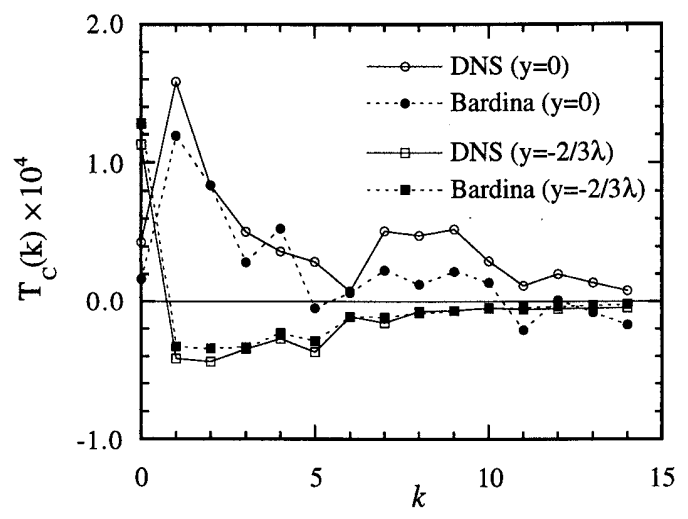

Fig. 13 GS-SGS energy transfer induced by the Cross term at $y=0$ and $y=-2 / 3 \lambda$ in a turbulent mixing layer edge $(y=-2 / 3 \lambda)$. Unlike in the case of homogeneous isotropic turbulence, the energy transfer induced by the Reynolds term in the turbulent mixing layer is comparable with that induced by the Leonard and Cross terms, which suggests that the Reynolds term is very important for the GS-SGS energy transfer in this turbulent flow. The Leonard and Cross terms give the opposite energy transfer far from the center of the shear layer, while this tendency is not observed in the center of the shear layer.

Figure 13 shows the exact energy transfer induced by the Cross term obtained from the DNS data and that obtained by use of the Bardina model at $y=$ 0 and $y=-2 / 3 \lambda$. As shown for homogeneous isotropic turbulence, the Bardina model represents the overall feature of the GS-SGS energy transfer. However, it is expected that, in the center of the shear layer, the energy transfer is smaller than that of obtained from the DNS data for the high wave number region, and negative energy transfer occurs for $k>10$. In the region far from the center of the shear layer, the energy transfer from SGS to GS is dominant. It is very important that the Bardina model can represent this negative energy transfer. Figures 14 and 15 show the energy transfer obtained by use of the Reynolds

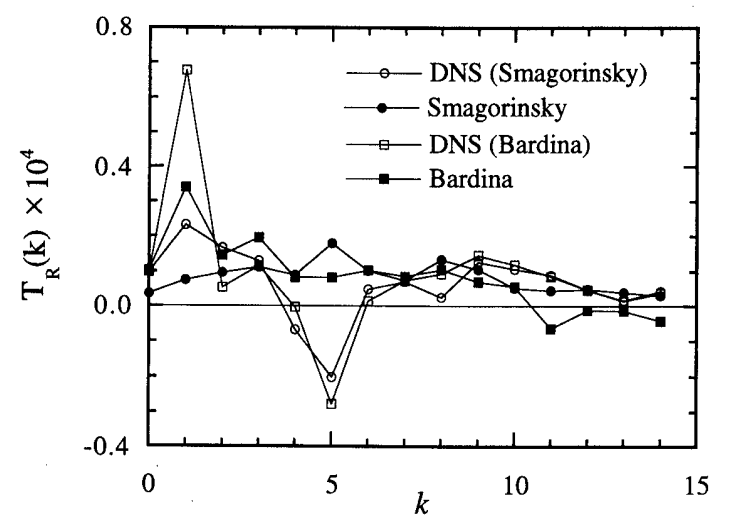

Fig. 14 GS-SGS energy transfer induced by the Reynolds term at $y=0$ in a turbulent mixing layer

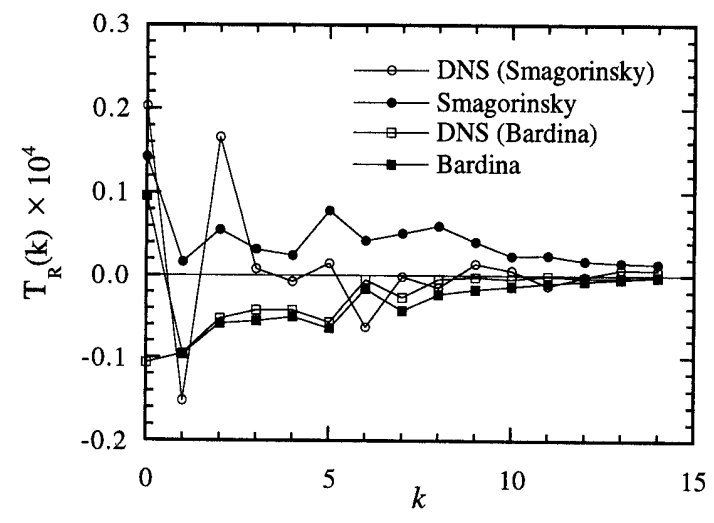

Fig. 15 GS-SGS energy transfer induced by the Reynolds term at $y=-2 / 3 \lambda$ in a turbulent mixing layer 
term at $y=0$ and $y=-2 / 3 \lambda$. In Fig. 14 , the reference energy transfer compared with that obtained by use of the Smagorinsky model is subtracted energy transfer due to $k_{g}$. In the Smagorinsky model, it is assumed that a mean shear exists in the turbulence and that the production of SGS energy is proportional to the dissipation. Therefore, compared to the case of homogeneous isotropic turbulence, the Smagorinsky model predicts the energy transfer relatively well in the center of the mixing layer. However, agreement between the energy transfers obtained by use of the Smagorinsky model and that obtained from DNS data becomes worse at the outer region of the mixing layer $(y=$ $-2 / 3 \lambda)$, where the magnitude of mean shear is low. The Bardina model for the Reynolds term predicts an inverse energy transfer in the high wave number region at the center of the shear layer, while the Bardina model gives a relatively good prediction of GS-SGS energy transfer through the mixing layer compared to that given by the Smagorinsky model.

Figure 16 shows the distribution of the GS-SGS energy transfer in the $y$ direction. Here, the solid

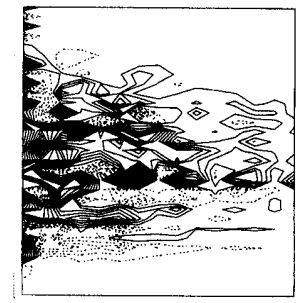

(a)

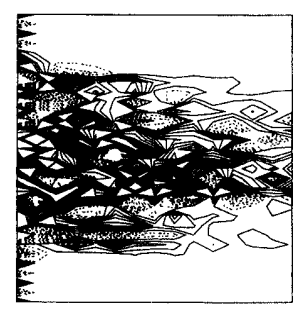

(c)

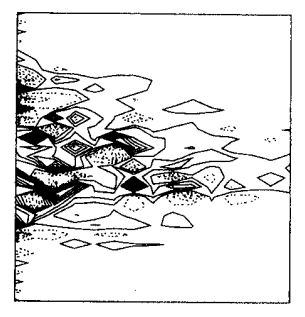

(e)

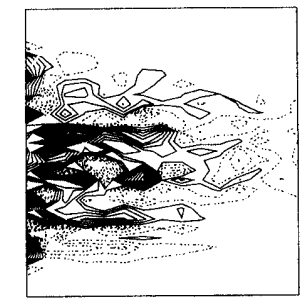

(b)

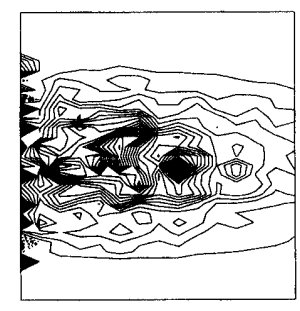

(d)

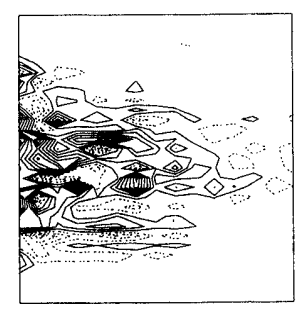

(f)
Fig. 16 Contour plots of GS-SGS energy transfer in the $y-k$ plane. ( $\mathrm{a}$ ) Cross term(DNS) ( $\mathrm{b}$ ) Cross term (Bardina model) ( c ) Reynolds term without $k_{g}$ (DNS) ( d ) Reynolds term (Smagorinsky model) ( e ) Reynolds term (DNS) ( f ) Reynolds term (Bardina model) lines and dotted lines represent positive and negative values, respectively. As stated above, the Bardina model for the Cross and Reynolds terms predicts the energy transfer relatively well at the middle wave number region in a shear layer, while the correlation in the high wave number region is low. As for the Smagorinsky model, it is only in the low wave number region that the structure of the energy transfer is reproduced. In the high wave number region, the structure of the energy transfer is not reproduced. In particular, there is no correlation between the Smagorinsky model and DNS data in the high wave number region.

As shown in the above discussion, the Bardina model for the Cross and Reynolds terms predicts an inverse energy transfer which may produce unphysical fluctuations in the high wave number region of the GS field. For investigation of this phenomenon, the energy transfer before the integration over the wave number should be discussed. Figure 17 shows the energy transfer at $y=0$ before the integration in twodimensional wave number space. A positive value

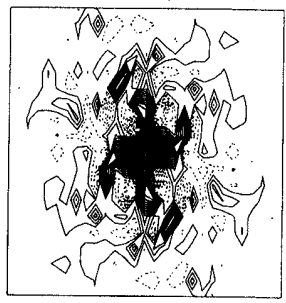

(a)

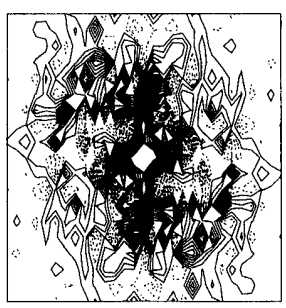

(c)

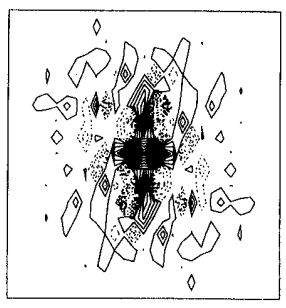

(e)

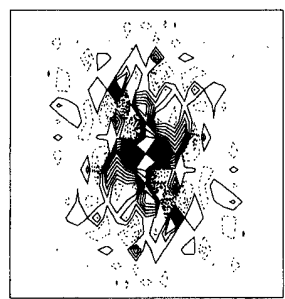

(b)

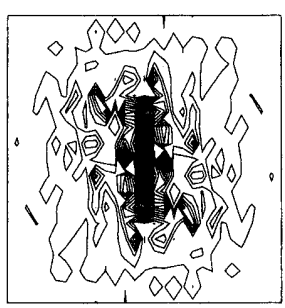

(d)

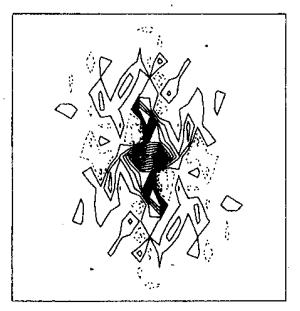

(f)
Fig. 17 Contour plots of GS-SGS energy transfer at $y=$ 0. ( a ) Cross term (DNS) ( b ) Cross term (Bardina model) (c) Reynolds term without $k_{g}$ (DNS) (d) Reynolds term (Smagorinsky model) (e ) Reynolds term(DNS) ( $\mathrm{f}$ ) Reynolds term (Bardina model) 


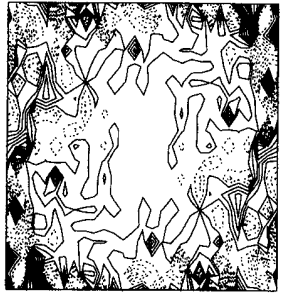

(a)

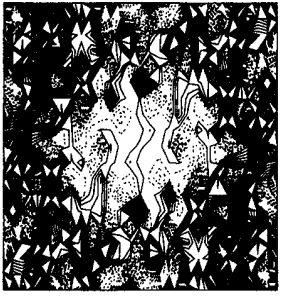

(c)

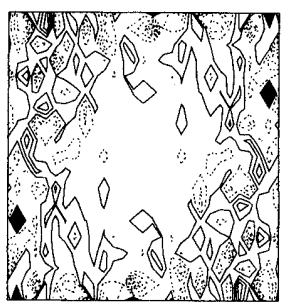

(e)

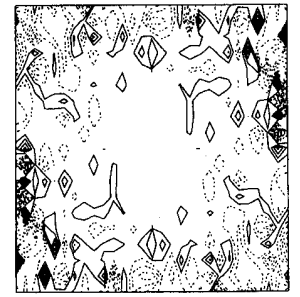

(b)

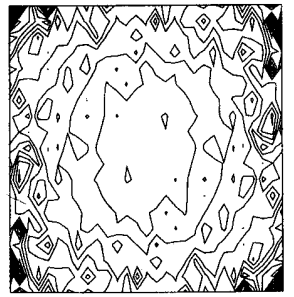

(d)

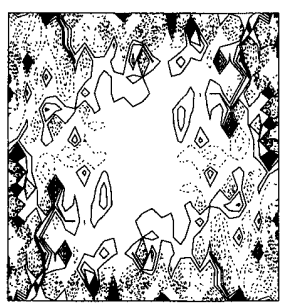

(f)
Fig. 18 Contour plots of normalized GS-SGS energy transfer at $y=0$. (a) Cross term(DNS) (b) Cross term (Bardina model) (c) Reynolds term without $k_{g}$ (DNS) (d ) Reynolds term (Smagorinsky model) (e ) Reynolds term(DNS) (f ) Reynolds term (Bardina model)

corresponds to forward scattering, and a negative one to backward scattering. From the DNS data, one can see that the SGS stresses include backward scattering. Note that the Smagorinsky model always predicts forward scattering due to the eddy viscosity representation. The Bardina models for the Cross and Reynolds terms predict the backward scattering very well in the low wave number region.

Figure 17 shows the energy transfer induced by the SGS term without any scaling, so that the energy transfer shows a large value in the low wave number region. Considering the magnitude of the GS energy, however, contribution of the GS-SGS energy transfer is significant in the high wave number region. Figure 18 shows the energy transfer at the center of the shear layer $(y=0)$ normalized by the GS energy of each wave number. From this normalization, we can understand the importance of the GS-SGS energy transfer to the GS energy transport. The exact energy transfers for the Cross and Reynolds terms show relatively large absolute values in the high wave number region. Therefore, small-scale motion in the
GS field is significantly affected by GS-SGS energy transfer. In the high wave number region, many wave number components show backward scattering. The energy transfer predicted by use of the Bardina model also represents backward scattering in the high wave number region. However, the absolute value of the backward scattering is too large. As a result, the total energy transfer shows a negative value.

\section{Conclusions}

GS-SGS energy transfer induced by SGS stress in large eddy simulation is investigated by direct numerical simulation of homogeneous isotropic turbulence and a turbulent mixing layer. From the results of this study, following conclusions were reached.

(1) No correlation between the Leonard and Cross terms exists from the viewpoint of energy transfer.

(2) The Smagorinsky model for the Reynolds term predicts very large energy transfer compared with that obtained from the DNS data.

(3) The Bardina models for the Reynolds and Cross terms predict large energy transfer from SGS to GS in the high wave number region, which indicates that the Bardina model causes excessive backward scattering in the high wave number region.

\section{Acknowledgment}

This work was supported by the Japan Ministry of Education, Science, Sports and Culture under Grant No. 05240108.

\section{References}

(1) Smagorinsky, J., Mon. Weather Rev., Vol. 91 (1963), p. 99.

(2) Bardina, J., Ferziger, J.H. and Reynolds, W.C., AIAA Paper, (1980) 80-1357.

( 3 ) Kraichnan, R.H., J. Atoms. Sci., Vol. 33 (1976), p. 1521.

(4) Chollet, J.P., Turbulent Shear Flows Vol. 4 (Bradbury, L.J.S., Durst, F., Launder, B.E., Schmidt, F.W. and Whitelaw, J.H. eds.), (1985), p. 66, Springer-Verlag, Berlin.

(5) Ohkitani, K. and Kida, S., Phys. Fluids, Vol. A 3 (1992), p. 794.

(6) Comte-Bellot, G. and Corssin, S., J. Fluid Mech., Vol. 48 (1971), p. 273.

(7) Tanahashi, M. and Miyauchi, T., Trans. Jpn. Soc. Mech. Eng., (in Japanese), Vol.60, No.579, B (1994), p. 3814.

(8) Horiuti, K., Phys. Fluids, Vol. A 1 (1989), p. 426.

(9) Miyauchi, T., Tanahashi, M. and Miyake, K., Trans. Jpn. Soc. Mech. Eng., (in Japanese), Vol. 61, No. 582, B(1995), p. 413.

(10) Horiuti, K., Phys. Fluids, Vol. A 5 (1993), p. 146.

(11) Lilly, D.K., NCAR Manuscript, (1966), p. 123. 